Thorax, 1980, 35, 881-883

\title{
Editorial
}

\section{Physician among surgeons: thoughts on preoperative assessment}

Some of my best friends are surgeons. A few drive fast cars, shoot birds, hunt foxes, write inadequate case notes, throw things when provoked and generally prefer action to thought-but an equal number of my physician friends do the same. Between physician and surgeon today there is surely no real difference of outlook or purpose. The old notion that physicians are conservative and surgeons radical no longer holds true; invasive investigations and modern drugs have seen to that. As one of my colleagues put it: "if they survive your tests, they'll stand my operation."

Why then in so many hospitals must we still live apart and meet only when something goes wrong? It is notoriously unwise to perforate in a medical ward or fibrillate in a surgical one. It is also true that when two patients with the same condition are referred one to a physician and the other to a surgeon, the former is more likely to get medicine and the latter surgery. If this is obvious, it is none the less disquieting for, when two such different alternatives exist, a rational decision can only be made by those who know the ultimate outcome of both. Neither physician nor surgeon alone can claim such knowledge but only the two together-not least because the failures of one tend to go to the other. The need for physicians and surgeons to live and work together is now recognised in many specialties and particularly our own. This editorial is appearing in the journal of a society dedicated to this concept, and I belong to a unit founded nearly 40 years ago upon the same principle by Morriston Davies and Robert Coope. Twenty-five years' experience with more than a dozen thoracic surgeons has convinced me that collaboration between physician and surgeon should begin before a final decision has been made about treatment, not after. All too familiar are physicians who sit on a patient until it is too late for surgery and surgeons who cry havoc about a postoperative disaster that could have been predicted if not avoided.

The physician can help in the preparations for an operation. With present day waiting lists, a patient may come in weeks or months after last Address for reprint requests: Dr Colin Ogilvie, Broadgreen Hospital,
Thomas Drive, Liverpool L14 3LB. attending a clinic with nothing between him and the final stroke but an unregistered houseman and a busy anaesthetist. This is especially likely to happen in hospitals operating the "hot bed" policy whereby the only way to protect a bed from the insatiable demands of a busy accident and emergency department is to have a patient waiting to leap into the bed of one ready for discharge. There is surely a case-and indeed this is already recognised in some centres-for a preoperative assessment clinic where physician, surgeon and anaesthetist can consult together. In such a place, there would be three questions to answer. (1) Is the treatment of choice surgical, medical, or both? (2) If surgical, is the patient fit? (3) If not, can he be made fit?

The answer to the first question can only be found if both physician and surgeon know what the other has to offer. In the old days, the physician was judge and the surgeon executioner. Later (and still in some centres today) each acted as both judge and executioner without reference to the other. Today, the two should surely together form a jury to decide whether one, the other, or both undertake the treatment of the patient.

The second question is one which is frequently put to a physician - usually because the houseman has found something wrong the day before the operation. Not all physicians understand the economic strategy of elective surgery: the need to bring together at a given hour a team of nurses, anaesthetists, surgeons, and technicians, a mass of equipment and, not least, the patient in a bed. The reluctance of a surgeon to put off an operation at the last moment is understandable but, more important, the patient may never find the courage to come back a second time. The physician must be sure of his ground before advising postponement but, being sure, he must stand firm.

Medical preoperative assessment is not just a matter of listening to the chest and looking at the ECG and lung function tests. The purpose of the operation must always be taken into account and, broadly speaking, there are two sorts: those which may make the patient worse for his future good and those which make him better for his present good. Examples in the first category are the removal of an otherwise healthy lung for cancer, 
parietal pleurectomy to prevent pneumothorax, and the closure of a symptomless atrial septal defect. In the second category come the decortication of a restricted lung, the excision of an emphysematous bulla, mitral valve replacement, and coronary by-pass surgery-operations which are carried out mainly for the relief rather than the prevention of symptoms. Patients in the first category may have to be convinced of the need for an operation while in the second it is sometimes the doctor who has to be convinced that the patient's symptoms merit surgery. In preoperative assessment, the physician must always ask not only whether the patient is good enough for surgery but also whether he is bad enough. To give a simple example of this apparent paradox: we may require an $\mathrm{FEV}_{1}$ or a test of exercise tolerance above a certain level before accepting a patient for pneumonectomy, since this usually worsens lung function, and below a certain level before we can justify the removal of an emphysematous bulla to improve lung function.

The physician should be prepared to share with the surgeon responsibility for weighing all the arguments for and against an operation. He must not content himself with merely predicting the likelihood of the patient's survival. He should also balance the risks and expectations of operating against the risks and expectations of not operating. This inevitably involves a careful review of the diagnosis and of the patient's principal complaints. When surgical excision of a lung tumour is contemplated in a patient with borderline function, for example, the most important question is not "What are the $\mathrm{FEV}_{1}$ and $\mathrm{PaCO}_{2}$ ?" but "Are we sure that this is cancer and that it is operable?" If it is, lung function would have to be poor indeed to justify withholding curative surgery. But if it could be a lung abscess, the risk of delaying removal of a cancer for a few weeks of antibiotic treatment may be less than the potential disaster of unnecessarily removing a simple lesion from a patient with inadequate lung function. Preoperative assessment must also include a review of the patient's chief symptoms. We cannot expect, as some patients coming forward for surgery in our own unit have done, that mitral valve replacement will improve chronic bronchitis nor that lobectomy for bronchiectasis will help dyspnoea.

Some attempt must be made to calculate the risk of a fatal or at least an unacceptable outcome to the operation. The physician should be aware of his own surgeon's mortality figures for these are obviously more relevant than the published reports of surgeons with results good enough to submit to a journal. For reasons already given, there is no critical value for any lung function measurement which determines in all circumstances whether thoracotomy is feasible. Certainly, no patient should be rejected for surgery on the basis of a single reading. An unfavourable result should invariably be repeated, preferably after appropriate treatment. In general, significant irreversible airflow obstruction as judged by persistent wheeze, a $\mathrm{FEV}_{1}$ less than $60 \%$ of the forced vital capacity after a bronchodilator, or a $\mathrm{PaCO}_{2}$ above $6.0 \mathrm{kPa}$ are adverse findings. Such patients are more likely than others to die of $\infty$ respiratory failure after operation and are less likely to benefit from mitral valve replacement or other operations designed to improve cardiopulmonary function. An important exception to this general rule is emphysematous bullae; these can cause airflow obstruction which is relieved by their removal.

The vital capacity, $\mathrm{PaO}_{2}$ and gas transfer factor (diffusing capacity) of ten give more information about the need for operation than its dangers. Considerable reduction in all three of these measurements with improvement after operation may occur especially in mitral valve disease. The vital capacity can be grossly impaired $\stackrel{\mathbb{Q}}{\Omega}$ by pulmonary collapse and also by thoracic cage $\overrightarrow{\vec{A}}$ deformities and patients with values of less than $\frac{3}{3}$ one litre have tolerated removal of a collapsed $\supset$ lung or fusion of a scoliotic spine without undue postoperative difficulties. Measurement of the ventilatory and circulatory responses to graded $\bar{O}$ exercise provides a reasonably objective test of disability and may sometimes help also to dis- $\frac{0}{7}$ tinguish cardiac from pulmonary causes. It is beyond the scope of this editorial to consider nonrespiratory aspects of preoperative assessment but it is clearly important to ensure that there is ade- 음 quate circulatory, renal, hepatic, haemopoietic, $D$ and metabolic function before going ahead with surgery.

If it has been decided that a patient may need but is unlikely to withstand thoracic surgery, the $N$ last question to answer is: can he be made fit $\omega$ enough? No final decision for or against surgery should be taken until the patient has been re- $\stackrel{\varrho}{C}$ assessed after the correction of all remediable $\stackrel{\mathscr{D}}{\mathscr{D}}$ defects. These include respiratory infections, + anaemia, cardiac failure or dysrhythmia, electro- 0 lyte imbalance, cigarette smoking, and reversible $\underset{\mathbb{D}}{\stackrel{D}{ }}$ airflow obstruction. Indeed, the treatment of such $\frac{\rho}{\mathbb{D}}$ conditions may reveal not just that the patient $\stackrel{\varrho}{\circ}$ can tolerate surgery but that, for the time being at least, he does not even need it. Examples of this 8 
include the resolution with antibiotics of a lung abscess thought to be malignant, the correction of anaemia improving angina in patients being considered for coronary by-pass surgery, and the relief of dyspnoea by adequate control of atrial fibrillation in mitral valve disease. Intensive bronchodilator therapy may likewise render an operation more safe or even unnecessary by improving breathlessness previously attributed to a potential surgical cause-for example, mitral disease or an emphysematous bulla. Failure to respond to a single dose of a bronchodilator during the course of routine lung function tests does not preclude improvement with more prolonged treatment.

The place and time for these preoperative procedures is of great importance. It is certainly not in a hospital bed the day before the operation (as happens only too often), nor even a week before, since none of the conditions we have considered can be safely or adequately treated in so short a time. On the other hand, to make this final assessment more than a month ahead of the operation leaves too much time for new complications to develop before the patient is admitted. This applies especially to labile asthmatic and bronchitic subjects in whom choice of season for an elective operation may also be relevant. Winter is usually best for atopic asthmatic patients and summer for bronchitic patients but this decision should be based on the past experience of the individual patient. It is clearly inappropriate to admit every patient to a hospital bed several weeks before an operation; apart from economic considerations, there are the dangers of cross-infection and thromboembolism. A reasonable programme of assessment for a planned cardiothoracic procedure (already carried out in some centres) would be to see the patient in an outpatient clinic three to four weeks before the appointed day. This would leave time to correct most remediable disorders, or alternatively to postpone the operation without this resulting in either a last minute disappointment for the patient or wasted theatre time. This would seem to be a most appropriate job for a physician and a good deal more rewarding in terms of potential benefit to the patient than many of the other tasks we are given today. If the surgeon can find time to be there too, so much the better.

COLIN OGILVIE

Royal Liverpool Hospital and Regional Cardiothoracic Centre Liverpool 